Address for Correspondence: Prof. John Y-J. Shyy, PhD, Department of Medicine, University of California, San Diego, 9500 Gilman Dr., La Jolla, CA 92093, USA.Email: jshyy@ucsd.edu

\begin{tabular}{|c|}
\hline Access this article 0 \\
\hline $\begin{array}{l}\text { Website: } \\
\text { www.intern-med.com }\end{array}$ \\
\hline $\begin{array}{l}\text { DOI: } \\
\text { 10.2478/jim-2018-0019 }\end{array}$ \\
\hline Quick Response Code: \\
\hline 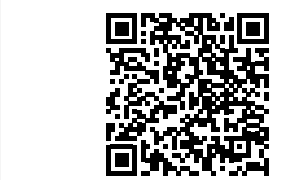 \\
\hline
\end{tabular}

\title{
Shear stress regulation of endothelium: A double-edged sword
}

\author{
Liang Bai ${ }^{1}$, John Y-J. Shyy ${ }^{1,2}$ \\ ${ }^{1}$ Cardiovascular Research Center, School of Basic Medical Sciences, Xi'an Jiaotong University Health Science \\ Center, Xi'an 710061, Shaanxi Province, China; \\ ${ }^{2}$ Department of Medicine/Division of Cardiology, University of California, San Diego, 9500 Gilman Dr, La Jolla,
} CA 92093, USA

\section{INTRODUCTION}

Vascular endothelium, the inner lining of the blood vessel wall, constantly responds to hemodynamic forces from blood flow. Thus, mechanical cues and the subsequent mechanotransduction in vascular endothelial cells (ECs) greatly affect vascular physiology and pathophysiology. ${ }^{[1-3]}$ The molecular mechanisms underlying the flow regulation of endothelial biology have been studied intensively by many labs including our own. In general, the blood flow pattern in the straight part of the arterial tree is less disturbed with high mean shear stress, which induces an array of atheroprotective genes [e.g., AMP-activated protein kinase (AMPK), Krüppel-like factor 4 (KLF4), Krüppel-like factor 2 (KLF2), and Sirtuin 1 (SIRT1)] in ECs. ${ }^{[4]}$ Conversely, blood flow in the curvatures and bifurcations of the arterial tree is disturbed with lower shear mean stress. A number of pro-inflammatory genes, such as monocyte chemoattractant protein-1 (MCP-1), sterol regulatory element binding protein 2 (SREBP2), NOD-like receptor family, pyrin domain containing protein 3 (NLRP3) and redox-regulated genes [e.g., NADPH oxidase (NOX)] are activated in ECs in these atheroprone areas, which results in dysfunctional endothelium. ${ }^{[8,9]}$ In this mini review, we summarize our recent findings in mechanotransduction and the corresponding responses in the endothelium, which result in atheroprotective versus atheroprone phenotype due to the respective atheroprotective or atheroprone flow patterns.

\section{ATHERO-PROTECTIVE FLOW REGULATION OF KLF4-CH25H/LXR AXIS}

KLF4 is a key transcription factor regulating the endothelial function promoting vascular homeostasis and conferring an atheroprotective phenotype both in vitro and in vivo. ${ }^{[10-12]}$ Laminar and/or pulsatile shear stress is potent stimuli to induce KLF4 in ECs. Consequent from KLF4 activation, the expression level of endothelial nitric oxide synthase (eNOS) and eNOS-derived NO bioavailability are elevated. ${ }^{[13]}$ At the upstream, the shear stress induction of KLF4 is mediated through a MEK5/ MEF2-dependent signaling pathway. ${ }^{[14,15]}$ Reports from several labs including our own indicate that shear stress regulates gene expression in ECs via epigenetic regulations including DNA methylation, chromatin remodeling, micro RNA, and long noncoding RNA (IncRNA). ${ }^{[16-22]}$ Pulsatile shear stress modifies histone H3K27 acetylation and H3 S10 phosphorylation in cultured ECs. ${ }^{[17,23]}$ Such epigenetic regulation leads to the increased expression of KLF4 and several KLF4 target genes including eNOS. By contrast, the oscillatory shear stress increases DNA methylation of CpG islands at the promoter region of the KLF4 gene, leading to suppressed expression of KLF4. ${ }^{[18]}$ Consistently, the disturbed flow increases the expression of DNA methyltransferase 3 a (DNMT3A) in swine aortic ECs, which in turn causes hypermethylation in the KLF4 promoter, thereby decreasing the binding of MEF2 to the promoter, resulting in suppressed KLF4 transcription. ${ }^{[18]}$ Interestingly, DNMT 
inhibitors revert the disturbed flow-suppressed KLF4, eNOS, and thrombomodulin, while increasing MCP-1. ${ }^{[18]}$

Recently, we showed that pulsatile shear stress increases the expression of cholesterol-25-hydroxylase (Ch25h) and liver X receptor (LXR) via KLF4 in ECs in vitro and in vivo. ${ }^{[17]}$ At the transcriptional level, KLF4 transactivates Ch25h and LXR via directly binding to the respective promoters (Figure 1). At the epigenetic level, the pulsatile shear stress decreased DNA methylation, but enhanced the modifications of active histone marks, namely, H3K 4 me3 and $\mathrm{H} 3 \mathrm{~K} 27 \mathrm{ac}$ in the upstream region of $\mathrm{Ch25h}$, which facilitated $\mathrm{Ch} 25 \mathrm{~h}$ induction. In vivo, the increased expression of $\mathrm{Ch} 25 \mathrm{~h}$ in the mouse thoracic aorta (atheroprotective flow area) was in line with the enriched H3K4me3 and H3K27ac but had attenuated DNA methylation. ${ }^{[17]}$ The pulsatile flow-induced KLF4-Ch25h/LXR axis contributed to an atheroprotective phenotype of endothelium, which was supported by increased atherosclerosis in the thoracic aorta of the Ch25h $\mathrm{h}^{-/} / \mathrm{ApoE}^{-/-}$double knockout mice fed a western diet. ${ }^{[17]}$

\section{ATHEROPRONE FLOW REGULATION OF SREBP2}

Disturbed flow pattern at arterial branches and curvatures causes inflammatory and oxidative responses in endothelium. These atherogenic events include the induction of interleukin $1 \beta$ (IL-1 $\beta$ ), NOX, MCP-1, vascular cell adhesion molecule-1 (VCAM-1), and intercellular adhesion molecule 1 (ICAM-1) etc., production of reactive oxygen species, endothelial dysfunction, and monocyte recruitment. ${ }^{[1,3]}$ Our recent study shows that the disturbed flow in vivo and oscillatory shear stress in vitro activate SREBP2, which in turn induces the NLRP3 inflammasome in ECs. ${ }^{[9]}$ Functioning as a transcription factor, SREBP2
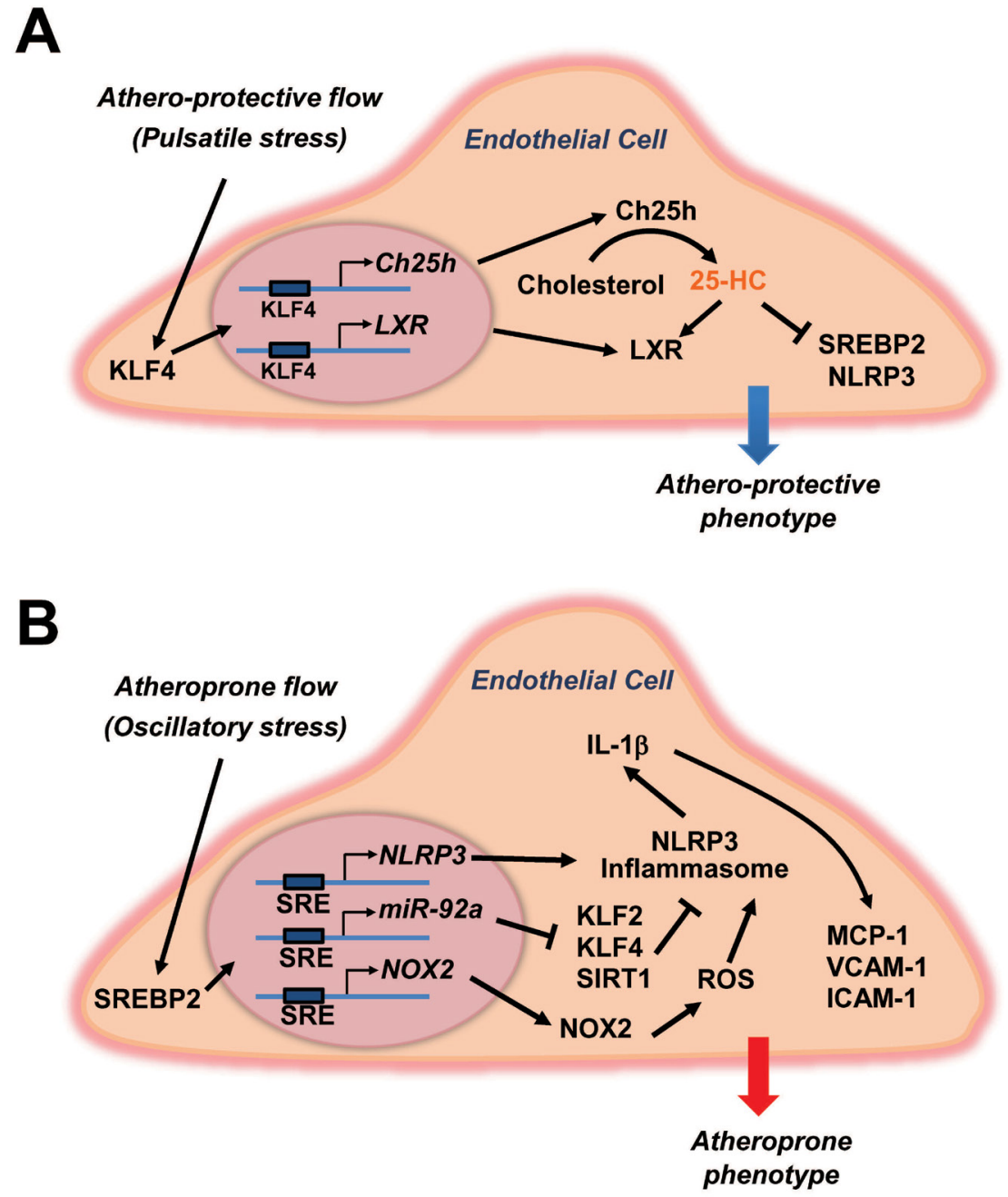

Figure 1: Shear stress regulation of endothelium. (A) Non-disturbed pulsatile flow induces KLF4-Ch25h/LXR axis. In endothelial cells, such shear stress induces KLF4, which in turn transactivates Ch25h and LXR, thus inhibiting SREBP2 and NLRP3 inflammasome and contributing to an athero-protective phenotype. (B) Disturbed and oscillatory flow induces SREBP2. Shear stress of this type activates SREBP2. SREBP2 then transactivates NLRP3, miR-92a, and NOX2. NLRP3 inflammasome activation causes IL-1ß production, while miR-92a targets KLF2, KLF4, and SIRT1. Oxidative stress is increased because of NOX2 induction. Together, these detrimental pathways lead to a dysfunctional endothelium, which presents an atheroprone phenotype. 
plays a key role in regulating cholesterol homeostasis. ${ }^{[2,25]}$ In addition to its canonical role in cholesterol biosynthesis, SREBP2 activates NLRP3 inflammasome in various cell types. ${ }^{[9,26]}$ As NLRP3 inflammasome activation leads to IL$1 \beta$ production, ${ }^{[27]}$ our results demonstrate that a disturbed flow-induced SREBP2 is sufficient and necessary for the increased innate immune responses in endothelium.

Micro RNA-33 (miR-33) is an intronic miR co-expressed with SREBP2. miR-33 targets mRNAs encoding ATPbinding cassette transporter A1 and G1 (ABCA1 and ABCG1) that are essential for reverse cholesterol transport (RCT). ${ }^{[28,29]}$ Intriguingly, LXR transactivates ABCA1 and ABCG1 to facilitate RCT. In hepatocytes and macrophages, cholesterol homeostasis is maintained through an intricate balance between the LXR-mediated RCT via ABCA1/ABCG1 and the SREBP2-mediated cholesterol biosynthesis. Our own research demonstrates that disturbed flow induces miR-33 in concert with the increased expression of SREBP2 in ECs. ${ }^{[9]}$ The impaired LXR-ABCA1/ABCG1 axis in ECs has been suggested to be pro-inflammatory in ECs. ${ }^{[17]}$ SREBP2 activation not only increases the level of miR-33, but also transactivates miR92a. ${ }^{[30]}$ It is well documented that miR-92a targets KLF2, KLF4, and SIRT1. ${ }^{[30,31]}$ These three molecules are crucial for endothelial homeostasis, in part via the induction of eNOS and the downregulation of IL-1 $\beta . .^{[4,13]}$

Thus, the disturbed flow-activated SREBP2 may impair the EC function through several mechanisms: (1) SREBP2 activation of NLRP3 inflammasome, resulting in increased production of IL-1 $\beta$; (2) miR-33 targeting ABCA1 and ABCG1 with consequent suppression of RCT; (3) miR-92a targeting KLF2, KLF4, and SIRT1, which leads to dysfunctional endothelium (Figure 1). In sum, these pathophysiological events contribute to an atheroprone phenotype of ECs. This thesis involving SREBP2 being a master regulator under a disturbed flow is strongly supported by the increased atherosclerosis seen in the thoracic aorta of an $A \mathrm{poE}^{-/-}$mouse line, in which the activated form of SREBP2 is overexpressed in the endothelium. ${ }^{[9]}$

\section{CONCLUSIONS}

In summary, several recent studies synergistically reveal that shear stress is a double-edged sword, depending upon the endothelial location in the arterial tree. Pulsatile, unidirectional flow activates the KLF4-Ch25h/ LXR homeostatic axis in ECs, thereby playing an atheroprotective role (Figure 1). By contrast, the disturbed flow activates SREBP2 with consequent induction of NLRP3 inflammasome, miR-33, and miR-92a in ECs. Disturbed flow being atheroprone, it increases innate immune response and decreases EC function (Figure 1). With respect to translational implication of these results, statins, the cholesterol lowering drug, activate the KLF4-Ch25h/LXR axis in endothelium ${ }^{[17]}$ and the Canakinumab Anti-inflammatory Thrombosis Outcome Study (CANTOS) uses anti-IL-1 $\beta$ to reduce the risk of cardiovascular events. ${ }^{[32]}$ The molecular basis underlying the common beneficial effect of cardiovascular medicine and shear stress may provide new therapeutic strategies for prevention and treatment of atherosclerosis.

\section{Source of Foundation}

The work is supported in part by the following funds: National Natural Science Foundation of China 81670452 and 91739112 (J.Y.S.) and China Postdoctoral Science Foundation 2016M590954 (L.B.).

\section{Conflict of Interests}

The authors declare no conflict of interests.

\section{REFERENCES}

1. Chiu JJ, Chien S. Effects of Disturbed Flow on Vascular Endothelium: Pathophysiological Basis and Clinical Perspectives. Physiol Rev 2011; 91:327-87.

2. Davies PF. Flow-Mediated Endothelial Mechanotransduction. Physiol Rev 1995; 75:519-60.

3. Davies PF. Hemodynamic shear stress and the endothelium in cardiovascular pathophysiology. Nat Clin Pract Cardiovasc Med 2009; 6:16-26.

4. Chen Z, Peng IC, Cui X, Li YS, Chien S, Shyy JY. Shear stress, SIRT1, and vascular homeostasis. Proc Natl Acad Sci U S A 2010; 107:10268-73.

5. Dekker RJ, van Thienen JV, Rohlena J, de Jager SC, Elderkamp YW, Seppen J, et al. Endothelial KLF2 links local arterial shear stress levels to the expression of vascular tone-regulating genes. Am J Pathol 2005;167:609-18.

6. Parmar KM, Larman HB, Dai GH, Zhang YH, Wang ET, Moorthy SN, et al. Integration of flow-dependent endothelial phenotypes by Kruppel-like factor 2. J Clin Invest 2006;116:49-58.

7. Zhang YJ, Lee TS, Kolb EM, Sun K, Lu X, Sladek FM, et al. AMP-activated protein kinase is involved in endothelial NO synthase activation in response to shear stress. Arterioscler Thromb Vasc Biol 2006; 26:1281-7.

8. Shyy YJ, Hsieh HJ, Usami S, Chien S. Fluid Shear-Stress Induces a Biphasic Response of Human Monocyte Chemotactic Protein-1 Gene-Expression in Vascular Endothelium. Proc Natl Acad Sci U S A 1994; 91:4678-82.

9. Xiao H, Lu M, Lin TY, Chen Z, Chen G, Wang WC, et al. Sterol Regulatory Element Binding Protein 2 Activation of NLRP3 Inflammasome in Endothelium Mediates Hemodynamic-Induced Atherosclerosis Susceptibility. Circulation 2013; 128:632-42.

10. Cowan CE, Kohler EE, Dugan TA, Mirza MK, Malik AB, Wary KK. Kruppel-Like Factor-4 Transcriptionally Regulates VE-Cadherin Expression and Endothelial Barrier Function. Circ Res 2010; 107:959-66.

11. Zhou GJ, Hamik A, Nayak L, Tian HM, Shi H, Lu Y, et al. Endothelial Kruppel-like factor 4 protects against atherothrombosis in mice. J Clin Invest 2012; 122:4727-31.

12. Yoshida T, Yamashita M, Horimai C, Hayashi M. Deletion of KruppelLike Factor 4 in Endothelial and Hematopoietic Cells Enhances Neointimal Formation Following Vascular Injury. J Am Heart Assoc 2014; 3:e000622.

13. Shen B, Smith RS, Hsu YT, Chao L, Chao J. Kruppel-like Factor 4 Is a 
Novel Mediator of Kallistatin in Inhibiting Endothelial Inflammation via Increased Endothelial Nitric-oxide Synthase Expression. J Biol Chem 2009; 284:35471-8.

14. Clark PR, Jensen TJ, Kluger MS, Morelock M, Hanidu A, Qi ZH, et al. MEK5 is Activated by Shear Stress, Activates ERK5 and Induces KLF4 to Modulate TNF Responses in Human Dermal Microvascular Endothelial Cells. Microcirculation 2011; 18:102-17.

15. Villarreal G Jr, Zhang Y, Larman HB, Gracia-Sancho J, Koo A, GarciaCardena G. Defining the regulation of KLF4 expression and its downstream transcriptional targets in vascular endothelial cells. Biochem Biophys Res Commun 2010; 391:984-9.

16. Marin T, Gongol B, Chen Z, Woo B, Subramaniam S, Chien S, et al. Mechanosensitive microRNAs-role in endothelial responses to shear stress and redox state. Free Radic Biol Med 2013; 64:61-8.

17. Li Z, Martin M, Zhang J, Huang HY, Bai L, Zhang J, et al. Kruppel-Like Factor 4 Regulation of Cholesterol-25-Hydroxylase and Liver X Receptor Mitigates Atherosclerosis Susceptibility. Circulation 2017; 136:1315-30.

18. Jiang YZ, Jimenez JM, Ou K, McCormick ME, Zhang LD, Davies PF. Hemodynamic Disturbed Flow Induces Differential DNA Methylation of Endothelial Kruppel-Like Factor 4 Promoter In Vitro and In Vivo. Circ Res 2014; 115:32-43.

19. Dunn J, Thabet S, Jo H. Flow-Dependent Epigenetic DNA Methylation in Endothelial Gene Expression and Atherosclerosis. Arterioscler Thromb Vasc Biol 2015; 35:1562-69.

20. Huang TS, Wang KC, Quon S, Nguyen P, Chang TY, Chen Z, et al. LINC00341 exerts an anti-inflammatory effect on endothelial cells by repressing VCAM1. Physiol Genomics 2017; 49:339-45.

21. Jiang YZ, Manduchi E, Jimenez JM, Davies PF. Endothelial Epigenetics in Biomechanical Stress Disturbed Flow-Mediated Epigenomic Plasticity In Vivo and In Vitro. Arterioscler Thromb Vasc Biol 2015 35:1317-26.

22. Hartung CT, Michalik KM, You XT, Chen W, Zeiher AM, Boon RA, et al. Regulation and Function of the Laminar Flow-induced Non-coding Rnas in Endothelial Cells. Circulation 2014; 130 (suppl 2):A18783.
23. Illi B, Nanni S, Scopece A, Farsetti A, Biglioli P, Capogrossi MC, et al. Shear stress-mediated chromatin remodeling provides molecular basis for flow-dependent regulation of gene expression. Circ Res 2003; 93:155-61.

24. Ikonen E. Cellular cholesterol trafficking and compartmentalization. Nat Rev Mol Cell Biol 2008; 9:125-38.

25. Brown MS, Goldstein JL. The SREBP pathway: Regulation of cholesterol metabolism by proteolysis of a membrane-bound transcription factor. Cell 1997; 89:331-40.

26. Chen Z, Martin M, Li Z, Shyy JY. Endothelial dysfunction: the role of sterol regulatory element-binding protein-induced NOD-like receptor family pyrin domain-containing protein 3 inflammasome in atherosclerosis. Curr Opin Lipidol 2014; 25:339-49.

27. Elliott EI, Sutterwala FS. Initiation and perpetuation of NLRP3 inflammasome activation and assembly. Immunol Rev 2015; 265:35-52.

28. Najafi-Shoushtari SH, Kristo F, Li YX, Shioda T, Cohen DE, Gerszten RE, et al. MicroRNA-33 and the SREBP Host Genes Cooperate to Control Cholesterol Homeostasis. Science 2010; 328:1566-9.

29. Rayner KJ, Suarez Y, Davalos A, Parathath S, Fitzgerald ML, Tamehiro N, et al. MiR-33 Contributes to the Regulation of Cholesterol Homeostasis. Science 2010; 328:1570-3.

30. Chen Z, Wen L, Martin M, Hsu CY, Fang LH, Lin FM, et al. Oxidative Stress Activates Endothelial Innate Immunity via Sterol Regulatory Element Binding Protein 2 (SREBP2) Transactivation of MicroRNA-92a. Circulation 2015; 131:805-14.

31. Fang Y, Davies PF. Site-Specific MicroRNA-92a Regulation of KruppelLike Factors 4 and 2 in Atherosusceptible Endothelium. Arterioscler Thromb Vasc Biol 2012; 32:979-87.

32. Ridker PM, Everett BM, Thuren T, MacFadyen JG, Chang WH, Ballantyne C, et al. Antiinflammatory Therapy with Canakinumab for Atherosclerotic Disease. N Engl J Med 2017; 377:1119-31.

How to cite this article: Bai L, Shyy JYL. Shear stress regulation of endothelium: A double-edged sword. J Transl Intern Med 2018; 6: 5861. 